\title{
COMPLETE PURE INJECTIVITY AND ENDOMORPHISM RINGS
}

\author{
J. L. GÓMEZ PARDO, NGUYEN V. DUNG, AND R. WISBAUER
}

(Communicated by Maurice Auslander)

\begin{abstract}
It is shown that if $M$ is a finitely presented completely pure injective object in a locally finitely generated Grothendieck category $\mathbf{C}$ such that $S=$ End $_{\mathbf{C}} M$ is von Neumann regular, then $S$ is semisimple. This is a generalized version of a well-known theorem of Osofsky, which includes also a result of Damiano on PCI-rings. As an application, we obtain a characterization of right hereditary rings with finitely presented injective hull.
\end{abstract}

In $[11,12]$ Osofsky showed that a ring, all of whose cyclic right modules are injective, is semisimple (Artinian). Faith [6] studied the structure of right PCI-rings, i.e., rings whose proper right cyclic modules are injective, and he left open the question of whether right PCI-rings must be right Noetherian. In [3] Damiano gave an affirmative answer to Faith's question. The key result in [3] was the fact that a proper cyclic finitely presented module $M_{R}$ over a right PCI-domain $R$ has a semisimple endomorphism ring $S$ [3, Proposition]. Damiano's proof uses the von Neumann regularity of $S$ that was observed earlier by Faith [6] and a modification of a constructive technique of Osofsky [12].

In this note we prove a general version for Grothendieck categories of Osofsky's theorem [11, 12], which includes also the above-mentioned result of Damiano. Furthermore, our arguments provide a simple proof of this result. We show that if $M$ is a finitely presented completely (pure) $M$-injective object in a locally finitely generated Grothendieck category $C$ such that $S=\operatorname{End}_{\mathbf{C}} M$ is von Neumann regular, then $S$ is semisimple. Consequently, if $M$ is a projective completely injective object in $\mathbf{C}$, then $M=\bigoplus_{i \in I} A_{i}$, where $\operatorname{End}_{\mathbf{C}} A_{i}$ are division rings and the subobjects of $A_{i}$ are linearly ordered. As an application to rings, we obtain a characterization of the right hereditary rings $R$ such that the injective hull $E\left(R_{R}\right)$ is finitely presented. This extends a result of Colby and Rutter [2]. Note that even in the module case, Damiano's arguments cannot be applied for proving our main theorem.

Let $\mathbf{C}$ be a Grothendieck category. An object $M$ of $\mathbf{C}$ is finitely presented if it is finitely generated and every epimorphism $A \rightarrow M$, where $A$ is finitely

Received by the editors June 20, 1991 and, in revised form, November 29, 1991.

1991 Mathematics Subject Classification. Primary 16D50, 16S50; Secondary 16D80, 16D90, $18 \mathrm{E} 15$

The first and second authors gratefully acknowledge the support of the Spanish Ministry of Education and Science (DGICYT PB87-0703). 
generated, has a finitely generated kernel. $\mathbf{C}$ is said to be locally finitely generated if it has a family of finitely generated generators. A short exact sequence $0 \rightarrow X \rightarrow Y \rightarrow Z \rightarrow 0$ in $\mathbf{C}$ will be called a pure sequence when the induced morphism $p: \operatorname{Hom}_{\mathbf{C}}(F, Y) \rightarrow \operatorname{Hom}_{\mathbf{C}}(F, Z)$ is an epimorphism for every finitely presented object $F$ of $\mathbf{C}$. In this case, $X$ is called a pure subobject of $Y$. An object $E$ of $\mathbf{C}$ is called pure injective when it has the injectivity property with respect to all pure sequences in $\mathrm{C}$ (cf. [16]). If $E$ and $M$ are objects of $\mathbf{C}$ and if $E$ is injective with respect to all pure sequences with middle term $M$, then we will say that $E$ is pure $M$-injective. An object $M$ is called completely injective (resp. completely pure $M$-injective) provided every quotient of $M$ is injective (resp. pure $M$-injective).

Clearly, an object $E$ is pure injective in $\mathbf{C}$ iff $E$ is pure $M$-injective for every object $M$ in $\mathbf{C}$. However, in some sense pure $M$-injective objects are far from pure injectivity. If $M$ is pure injective in $C$ and $S=\operatorname{End}_{\mathbf{C}} M$, then it is well known that $S / J(S)$ is von Neumann regular (see, e.g., [16, Corollary 1.6]). Suppose that $R$ is a right noetherian ring. Then it is easy to see that a right ideal $A$ of $R$ is a pure submodule of $R_{R}$ iff $A$ is a direct summand of $R_{R}$. Thus every right $R$-module (in particular, $R_{R}$ ) is pure $R$-injective. But indeed $R / J(R)$ need not be a von Neumann regular ring.

Now we are ready to prove our main result.

Theorem 1. Let $\mathbf{C}$ be a locally finitely generated Grothendieck category and $M$ a finitely presented object of $\mathbf{C}$ which is completely pure $M$-injective and has a von Neumann regular endomorphism ring $S$. Then $S$ is a semisimple ring.

Proof. Using Osofsky's theorem [11, 12], it will be enough to prove that each cyclic right $S$-module is injective. By [9, Theorem VI.3.1], the functor $\operatorname{Hom}_{\mathbf{C}}(M,-)$ from $\mathbf{C}$ to Mod-S has a left adjoint, which we denote by $-\otimes_{S} M:$ Mod- $S \rightarrow \mathbf{C}$, and it is not difficult to check that $S \otimes_{S} M \cong M$ canonically. Let $f: K \rightarrow C$ be an $S$-homomorphism from a right ideal $K$ of $S$ to a cyclic right $S$-module $C$. We must show that $f$ has an extension to a homomorphism $g: S \rightarrow C$. We may write $K=\lim K_{i}$, where $\left\{K_{i}\right\}_{I}$ is the direct system of all the finitely generated right ideals of $S$ contained in $K$, and, similarly, $C=\lim C_{j}$, where the $C_{j}$ 's are finitely presented cyclic right $S$-modules. Since $S$ is von Neumann regular, the $K_{i}$ 's and the $C_{j}$ 's are direct summands of $S_{S}$. Thus, since $-\otimes_{S} M$ is an additive functor, we have that for each $i \in I$ and $j \in J, K_{i} \otimes_{S} M$ and $C_{j} \otimes_{S} M$ are isomorphic to direct summands of $M$. Clearly, the adjunction morphisms

$$
K_{i} \rightarrow \operatorname{Hom}_{\mathbf{C}}\left(M, K_{i} \otimes_{S} M\right), \quad C_{j} \rightarrow \operatorname{Hom}_{\mathbf{C}}\left(M, C_{j} \otimes_{S} M\right)
$$

are isomorphisms. The functor $-\otimes_{S} M$, being a left adjoint, preserves colimits and, in particular, direct limits. Also, since $M$ is a finitely presented object of $\mathbf{C}$, the functor $\operatorname{Hom}_{\mathbf{C}}(M,-)$ preserves direct limits (see [17, Proposition V.3.4]). Thus we see that the canonical homomorphisms

$$
K \rightarrow \operatorname{Hom}_{\mathbf{C}}\left(M, K \otimes_{S} M\right), \quad C \rightarrow \operatorname{Hom}_{\mathbf{C}}\left(M, C \otimes_{S} M\right)
$$

are isomorphisms, and from this it follows that $f$ can be identified with the homomorphism $\operatorname{Hom}_{\mathbf{C}}\left(M, f \otimes_{S} M\right)$.

On the other hand, if we take an epimorphism $S \rightarrow C \rightarrow 0$ in Mod-S, we see that since $-\otimes_{S} M$ is right exact, $C \otimes_{S} M$ is a quotient object of $M$ in $\mathbf{C}$ 
and hence is pure $M$-injective. The induced morphism $K \otimes_{S} M \rightarrow S \otimes_{S} M \cong M$ is the direct limit of the split monomorphisms $K_{i} \otimes_{S} M \rightarrow S \otimes_{S} M$ and hence is a pure monomophism (see, e.g., [19, 33.8]). Thus we have in $\mathbf{C}$ a diagram with exact row

$$
\begin{aligned}
0 \rightarrow & K \otimes_{S} M \rightarrow S \otimes_{S} M \cong M \\
& f \otimes_{S} M \downarrow / h \\
& C \otimes_{S} M
\end{aligned}
$$

which can be completed by the pure $M$-injectivity of $C \otimes_{S} M$. By applying the functor $\operatorname{Hom}_{\mathbf{C}}(M,-)$ to this diagram, we obtain the $S$-homomorphism $\operatorname{Hom}_{\mathbf{C}}(M, h): S \rightarrow C$ which extends $f: K \rightarrow C$. This shows that $C$ is indeed an injective right $S$-module.

Remarks. (a) It is easy to see that, in the above proof, $C \otimes_{S} M$ is a quotient of $M$ by a pure submodule. Thus Theorem 1 remains true if we merely assume that every quotient of $M$ by a pure subobject is pure $M$-injective. There is a good supply of modules satisfying this condition; for example, all the pure injective modules over a ring of right pure global dimension $\leq 1$ (and, in particular, over any countable ring [7, Théorème 7.10]). Observe also that, in fact, the same arguments show that for a finitely presented object $M$ satisfying the above condition, every flat cyclic right $S$-module is pure $S$-injective (without assuming that $S$ is regular).

(b) It is natural to ask whether the finitely presented condition of $M$ in Theorem 1 could be weakened. In particular, it would be interesting to know if Theorem 1 still remains valid when $M$ is finitely generated. We remark that, in fact, the above arguments will work if $M$ is assumed to be finitely generated and the functor $\operatorname{Hom}_{\mathbf{C}}(M,-)$ preserves direct limits of objects which are isomorphic to direct summands of $M$.

Corollary 2. Let $\mathbf{C}$ be a locally finitely generated Grothendieck category and $M$ a finitely generated projective completely injective object in $\mathbf{C}$. Then $S=$ End $_{\mathbf{C}} M$ is semisimple.

Proof. Clearly $M$ is finitely presented. Let $f$ be any element in $S$. Then $\operatorname{Im}(f)$ is injective and hence is a direct summand of $M$. It follows that $\operatorname{Im}(f)$ is projective, so $\operatorname{Ker}(f)$ is a direct summand of $M$. Thus $S$ is a von Neumann regular ring (see, e.g., [17, exercise 10, p. 110]). By Theorem 1, $S$ is semisimple.

The next result is well known for modules over a ring (see, e.g., [8, Corollary 13.6.7]).

Lemma 3. Let $M$ be a projective injective object in a locally finitely generated Grothendieck category C. Then $M$ is a coproduct of finitely generated objects.

Proof. A categorical version of Kaplansky's theorem on projective modules was proved in [13, Lemma 3.8]. Using this, the module-theoretic arguments of [8, Corollary 13.6.7] may be carried over verbatim.

Corollary 4. Let $\mathbf{C}$ be a locally finitely generated Grothendieck category and $M$ a projective completely injective object in $\mathbf{C}$. Then $M=\bigoplus_{i \in I} A_{i}$, where for each $i \in I, S_{i}=$ End $_{\mathbf{C}} A_{i}$ is a division ring and the subobjects of $A_{i}$ are linearly ordered. 
Proof. By Lemma 3, $M=\bigoplus_{j \in J} M_{j}$, where each $M_{j}$ is finitely generated. By Corollary 2, $\operatorname{End}_{\mathbf{C}} M_{j}$ is semisimple, hence $M_{j}$ is a finite direct sum of indecomposable subobjects (see, e.g., [17, Proposition XIV.1.7]). Therefore, we have $M=\bigoplus_{i \in I} A_{i}$, where each $A_{i}$ is an indecomposable object. Clearly $S_{i}=$ End $_{\mathrm{C}} A_{i}$ is a division ring, which implies that $A_{i}$ has a unique maximal subobject $K_{i}$ containing every proper subobject of $A_{i}$ (e.g., $\left.[19,19.7]\right)$. Now a standard argument, similar to the proof of [14, Proposition 3], shows that the subobjects of $A_{i}$ are linearly ordered.

Let $R$ be an associative ring with identity and $M_{R}$ a unitary right $R$ module. Denote by $\sigma[M]$ the full subcategory of Mod- $R$ whose objects are submodules of $M$-generated modules. Then $\sigma[M]$ is a locally finitely generated Grothendieck category and Theorem 1 can be applied.

Corollary 5. Let $M_{R}$ be a completely pure $M$-injective module which is finitely presented in $\sigma[M]$ and has a von Neumann regular endomorphism ring $S$. Then $S$ is semisimple.

Note that a module $M_{R}$ which is finitely presented in $\sigma[M]$ need not be finitely presented in Mod- $R$; for instance, any finitely generated self-projective module (see [19] for the definition) is finitely presented in $\sigma[M]$. In particular, any simple module $M$ is finitely presented in $\sigma[M]$, but, obviously, it need not be finitely presented in Mod- $R$. We also have natural interpretations of Corollaries 2 and 4 in $\sigma[M]$, with $M$ being completely $M$-injective and finitely generated quasi-projective or $\Sigma$-quasi-projective (i.e., projective in $\sigma[M])$, respectively. These extend [4, Corollary 5], where the semisimplicity of $S=\operatorname{End}\left(M_{R}\right)$ was obtained under the stronger hypotheses that $M_{R}$ is (cyclic) quasi-projective and every cyclic module in $\sigma[M]$ is $M$-injective.

Finally, we apply our main theorem to hereditary rings. In [2, Theorem 3.2], Colby and Rutter showed that $R$ is a right hereditary ring with $E\left(R_{R}\right)$ projective iff $R$ is a (two-sided) hereditary Artinian QF-3 ring. Clearly, if $R$ is a ring such that $E\left(R_{R}\right)$ is projective, then $E\left(R_{R}\right)$ is finitely generated and hence finitely presented (see, e.g., [8, Lemma 13.6.6]). Thus our next result may be regarded as an extension of Colby-Rutter's theorem.

Corollary 6. The following conditions are equivalent for a ring $R$ :

(1) $R$ is right hereditary and $E\left(R_{R}\right)$ is finitely presented.

(2) $R$ is right hereditary right Artinian and every injective right $R$-module is a direct sum of finitely generated modules.

(3) $R$ is a right hereditary right Artinian ring with Morita duality.

Proof. (1) $\Rightarrow(2)$. Clearly $R$ is right nonsingular, hence $S=\operatorname{End}\left(E\left(R_{R}\right)\right)$ is von Neumann regular (e.g., [17, Theorem XIV.1.2]). Also, $E\left(R_{R}\right)$ is completely injective, so by Corollary $5, S$ is semisimple. It follows that $E\left(R_{R}\right)$, and hence $R_{R}$, have finite uniform dimension. By [15, Corollary 2], $R$ is right Noetherian. Since $E\left(R_{R}\right)$ is finitely generated, it follows that $R$ is right Artinian by $[18$, Theorem A]. Let $C \cong R / A$ be any cyclic right $R$-module. Then clearly $E\left(R_{R}\right) / A$ is injective and finitely generated. Thus $C$, being contained in $E\left(R_{R}\right) / A$, has a finitely generated injective hull. Now let $M_{R}$ be any injective module. Then $M_{R}=\bigoplus_{i \in I} N_{i}$, where each $N_{i}$ is indecomposable injective. So $N_{i}$ is uniform and hence is the injective hull of any nonzero cyclic submodule $C \subseteq N_{i}$. This shows that $N_{i}$ is finitely generated for each $i \in I$. 
$(2) \Rightarrow(3)$. This follows from the well-known fact that a right Artinian ring $R$ has Morita duality iff the injective hull of each simple right $R$-module is finitely generated (see, e.g., $[19,47.15])$.

$(3) \Rightarrow(1)$. This is clear.

Remarks. (a) In the recent work [14], Osofsky and Smith proved a general theorem on cyclic completely $C S$-modules from which they obtained as a corollary the fact that right PCI-rings are right Noetherian. Corollary 2 was also obtained in [5] (for modules), by adapting the techniques of [14]. However, there are apparently no direct relationships between our Theorem 1 and the Osofsky-Smith theorem [14].

(b) The rings satisfying Corollary 6 need not be QF-3. An example of this can be found in [1, p. 353] if the division ring $\Delta$ from that example is, furthermore, assumed to be finite. However, by [10, Corollary 3.7], a right hereditary ring $R$ with $E\left(R_{R}\right)$ cyclic finitely presented is semisimple. On the other hand, we do not know if Corollary 6 still remains true if the ring $R$ is right hereditary and $E\left(R_{R}\right)$ is finitely generated.

\section{ACKNOWLEDGMENT}

The authors would like to thank the referee for several helpful suggestions.

\section{REFERENCES}

1. V. C. Cateforis and F. L. Sandomierski, On modules of singular submodule zero, Canad. J. Math. 23 (1971), 345-354.

2. R. R. Colby and E. A. Rutter, Generalizations of QF-3 algebras, Trans. Amer. Math. Soc. 153 (1971), 371-386.

3. R. F. Damiano, A right PCI-ring is right Noetherian, Proc. Amer. Math. Soc. 77 (1979), 11-14.

4. Dinh Van Huynh and R. Wisbauer, A characterization of locally Artinian modules, J. Algebra 132 (1990), 287-293.

5. __ Self-projective modules with $\pi$-injective factor modules, J. Algebra (to appear).

6. C. Faith, When are proper cyclics injective?, Pacific J. Math. 45 (1973), 97-112.

7. L. Gruson and C. U. Jensen, Dimensions cohomologiques relieés aux foncteurs $\lim ^{(i)}$, Lecture Notes in Math., vol. 867, Springer-Verlag, Berlin and New York, 1981, pp. 234294.

8. F. Kasch, Modules and rings, Academic Press, London, 1982.

9. B. Mitchell, Theory of categories, Academic Press, New York, 1965.

10. Nguyen V. Dung, A note on hereditary rings or non-singular rings with chain condition, Math. Scand. 66 (1990), 301-306.

11. B. L. Osofsky, Rings all of whose finitely generated modules are injective, Pacific J. Math. 14 (1964), 645-650.

12. __ Noninjective cyclic modules, Proc. Amer. Math. Soc. 19 (1968), 1383-1384.

13. $\ldots$, Projective dimension of 'nice' directed unions, J. Pure. Appl. Algebra 13 (1978), 179219.

14. B. L. Osofsky and P. F. Smith, Cyclic modules whose quotients have all complement submodules direct summands, J. Algebra 139 (1991), 342-354.

15. F. L. Sandomierski, Nonsingular rings, Proc. Amer. Math. Soc. 19 (1968), 225-230.

16. D. Simson, On pure semi-simple Grothendieck categories, Fund. Math. 100 (1978), 211-222.

17. B. Stenström, Rings of quotients, Springer-Verlag, Berlin and New York, 1975. 
18. C. I. Vinsonhaler, Orders in QF-3 rings, Suppl. J. Algebra 17 (1971), 149-151.

19. R. Wisbauer, Foundations of module and ring theory, Gordon and Breach, Reading, MA, 1991. SPAIN

Departamento de Matemáticas, Universidad de Murcia, 30100 Espinardo, Murcia,

Current address, J. L. Gómez Pardo: Departamento de Alxebra, Facultade de Matematicas, Universidade de Santiago, 15771 Santiago, Spain

E-mail address, J. L. Gómez Pardo: jlgpardo@seins.usc.es

Institute of Mathematics, P.O. Box 631 Bo Ho, Hanoi, Vietnam

Mathematisches Institut der Universität DüsSeldorf, 4000 DÜSSeldorf, Federal REPUBLIC OF GERMANY

E-mail address, R. Wisbauer: wisbauer@rz.ze8.de 